\title{
Experience and Technique for Zenker's Diverticulum Per Oral Endoscopic Myotomy: Z-POEM
}

\author{
Eitan Podgaetz ${ }^{1}$ Vani Konda ${ }^{2}$ \\ ${ }^{1}$ Center for Thoracic Surgery, Baylor Scott \& White North Texas, \\ Dallas, Texas, United States \\ ${ }^{2}$ Center for Esophageal Diseases, Baylor University Medical Center at \\ Dallas, Dallas, Texas, United States
}

\begin{abstract}
Address for correspondence Eitan Podgaetz, MD, MPH, FACS, Center for Thoracic Surgery, Baylor Scott \& White North Texas, 3410 Worth St, suite 325, Dallas, TX 75246, United States (e-mail: eitan.podgaetz@bswhealth.org).
\end{abstract}

Thorac Cardiovasc Surg 2021;69:228-230.

\begin{abstract}
Keywords

- cricopharyngeal muscle

- Zenker's diverticulum

- per oral myotomy

Objective With the advent of minimally invasive surgery, incisionless surgery, and third-space endoscopy, the treatment for Zenker's diverticulum has also moved toward less invasive techniques

Methods New incisionless per oral techniques can be applied for cricopharyngeal myotomy in Zenker's diverticulum.

Results Five patients underwent Zenker's diverticulum per oral endoscopic myotomy (Z-POEM) without complications, minimal discomfort, and narcotic consumption, with complete resolution of their symptoms by history and Eckardt scores.

Conclusions Z-POEM is performed entirely endoscopically with very little associated pain or complication rates, with short-term follow-up having excellent functional and symptomatic results.
\end{abstract}

\section{Introduction}

Zenker's diverticulum (ZD) is a saclike outpouching in the hypopharyngeal area just above the cricopharyngeal (CP) muscle. $\mathrm{ZD}$ is a rare disease that occurs in approximately $0.1 \%$ of the population, and its incidence varies widely by gender, race, and geographic location. ZD was actually first described by Abraham Lodlow in $1769{ }^{1}$

While some cases may be asymptomatic, symptoms include dysphagia, halitosis, choking, and coughing. Aspiration is a known complication. The treatment of ZD has evolved over time to a minimally invasive approach including rigid and flexible endoscopic techniques with both diverticular septal division and stapling. Endoscopic techniques were first attempted by Mosher in 1917 by dividing the $\mathrm{CP}$ muscle. ${ }^{2}$ Since then, the $\mathrm{CP}$ muscle has been divided with scissors, diathermy, $\mathrm{CO}_{2}$ laser, KTP (potassium titanyl phosphate) laser, papillotomes, and argon plasma coagulation. ${ }^{3}$

A diverticular stapling technique described in Belgium and England in $1993^{4}$ is now utilized with a specialized double spreading laryngoscope (Weerda) ${ }^{5}$ where an automatic surgical stapler seals and divides the $\mathrm{CP}$ at the same time.

Other flexible endoscopic techniques have also been described. ${ }^{6}$ Third-space endoscopy and per oral endoscopic techniques can be used to approach the $\mathrm{CP}$ muscle.

\section{Methods}

This is a retrospective single-institution study conducted from January 2019 to February 2020. This study was approved by our Institutional Review Board. Consecutive patients undergoing Zenker's diverticulum per oral received

July 14,2020

accepted after revision

August 21, 2020

published online

October 21, 2020
DOI https://doi.org/ 10.1055/s-0040-1717127. ISSN 0171-6425.

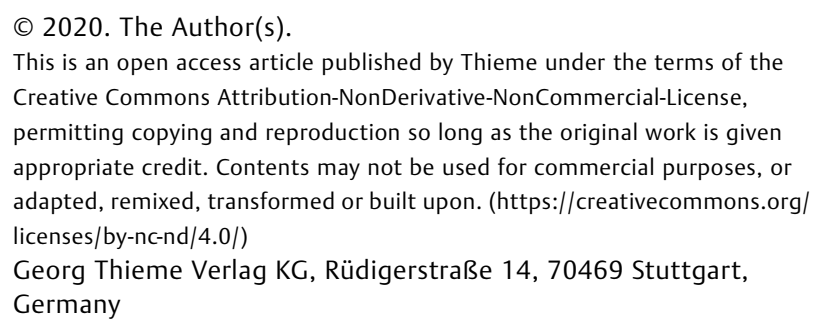




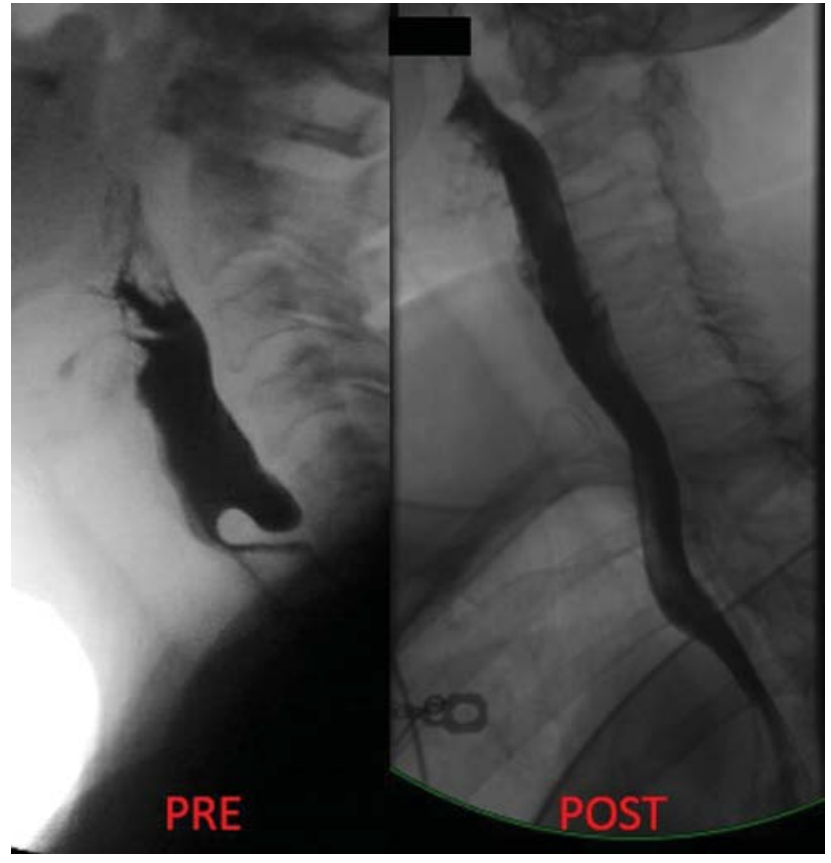

Fig. 1 Lateral view of preoperative and postoperative esophagram with complete elimination of the diverticulum after Zenker's diverticulum per oral endoscopic myotomy.

endoscopic myotomy (Z-POEM) were included. ZD was diagnosed with both endoscopy and radiological studies (-Fig. 1A), and all patients were symptomatic from the ZD.

Dysphagia Eckardt scores were used during preoperative visit and postoperatively.

Exclusion criteria included previous surgical treatments for the ZD.

\section{Technique Description}

Procedure is performed under general anesthesia with rapid sequence endotracheal intubation with a 7.0 endotracheal tube in the left lateral position.

A regular adult gastroscope (GIF-HQ 190/GIF-H180J, Olympus, Center Valley, PA) with a straight disposable distal attachment clear cap (D-201-11804, Olympus) is used.

Only carbon dioxide is used for insufflation at a low flow of 2 to $3 \mathrm{~L} / \mathrm{min}$ and not air as some degree of subcutaneous emphysema and potentially pneumothorax can occur.

We give a second-generation cephalosporin, an antifungal, and a proton-pump inhibitor at the initiation of the procedure.

Endoscopy is performed, the entire esophagus is examined, the $\mathrm{ZD}$ is then identified, and the $\mathrm{CP}$ bar is seen ( - Fig. 2A). If any residual debris is found on the diverticulum, it is washed and cleaned.

Using diluted methylene blue saline solution without epinephrine, a submucosal bleb is made with an endoscopic needle (Interject 23Ga, Boston Scientific, Spencer, IN). Only a few milliliters are required $(2-5 \mathrm{~mL})$ for this initial submucosal bleb as larger amounts will obscure the view altogether.

We use a HybridKnife I-Type I-Jet (Erbe, Tubingen, Germany). The initial 1.5 - to 2 -cm vertical mucosotomy

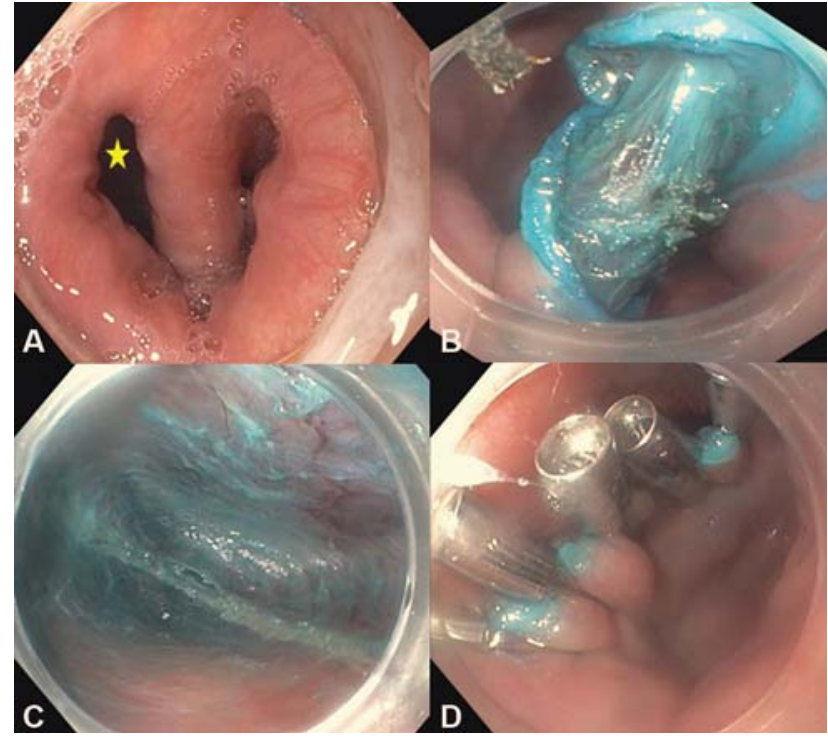

Fig. 2 (A) Endoscopic view of Zenker's diverticulum. Yellow star indicates the diverticular side. (B) Cricopharyngeal muscle exposed after tunneling. (C) Complete myotomy. (D) Clip closure of mucosal edges.

incision is made along the long axis of the septum using Endocut I Effect 3, Cut Duration 2, Cut Interval 2 (Erbe).

Careful endoscopic submucosal dissection is performed until the apex of the CP muscle is identified. We then separate the submucosal space between the $\mathrm{CP}$ and the diverticulum and between the $\mathrm{CP}$ and the esophageal wall until the submucosal plane is completely developed on both sides of the CP. For the submucosal dissection, we use Swift coagulation mode 30 Watts Effect 3. When large vessels were identified, these were treated using the Coagrasper (FD-410LR, Olympus) in soft coagulation mode at $80 \mathrm{~W}$ on Effect 5.

The esophageal mucosa is preserved in its entirety. The submucosal tunnel is then completed until we reach past the end on the diverticulum on one side and we dissect 1 to $2 \mathrm{~cm}$ distal to the end of the $\mathrm{CP}$ muscle on the esophageal side.

At the completion of the tunneling, the $\mathrm{CP}$ septum is entirely exposed (-Fig. 2B) and then divided. Special attention is paid to prevent inadvertent mucosal injury as we divide the septum. The septotomy is extended past the base of the septum, and esophageal myotomy is extended for 1 $2 \mathrm{~cm}$ to ensure complete division (-Fig. 2C). Then the mucosotomy is closed with endoscopic 11-mm clips (-Fig. 2D).

\section{Postoperative Management}

Patients are admitted overnight and kept nil per os with intravenous (IV) fluids, IV antibiotics, and scheduled antiemetics. A video esophagram is preformed the next morning to look for contrast extravasation.

Patients are then started on a 3-3-3 diet consisting of 3 days of clear liquids, 3 days of full liquids, and then 3 weeks of a soft diet. All patients are discharged with a week of liquid oral antibiotics. 
All patients are seen in the clinic for a follow-up visit in 1 to 2 weeks with a chest X-ray looking for pneumomediastinum or pleural effusion and then again in 3 months with a repeat esophagram.

\section{Results}

A total of five patients (three males and two females) with a median age of 63 years (range: 57-74 years) underwent per oral CP myotomy.

All patients had dysphagia, and $80 \%$ of patients had regurgitation, aspiration, chronic cough, inability to sleep flat, and halitosis. The median diverticular size was $3 \times 2.2 \mathrm{~cm}$ (ranging from 1 to $5.5 \mathrm{~cm}$ craniocaudally to 0.6 to $4.7 \mathrm{~cm}$ transverse).

The Z-POEM median procedure duration time was 74 minutes (range: 57-123 minutes). Technical success was $100 \%$. There were no perioperative complications, and all patients were discharged home the following morning after a video esophagram.

All patients received postoperative intravenous acetaminophen, and only two patients received postoperative narcotics for a median of 3 morphine milligram equivalents per day.

On their first postoperative visit, all patients reported complete symptomatic relief of their preoperative symptoms with an Eckardt score of 0 (preoperative Eckardt score median: 4.4), and this has persisted for a median follow-up of 5 months.

Postoperative esophagrams also demonstrate radiological improvement and in some cases complete elimination of the diverticulum despite not performing a diverticulectomy (-Fig. 1B).

\section{Comment}

Our study is unique as it represents a small series of successful Z-POEM performed by a thoracic surgeon in collaboration with a gastroenterologist in a multidiciplinary esophageal center. Third-space endoscopy is rapidly becoming a viable alternative to open or minimally invasive surgical techniques. Our early results support that Z-POEM is a safe and feasible procedure.

There is limited literature on per oral endoscopic technique for the treatment of $\mathrm{ZD}$ in the form of case reports or retrospective experiences in mostly gastroenterology publications. $^{7,8}$

Some of the criticisms of the transoral stapled technique is that the stapler often does not incorporate the whole CP muscle and may leave some muscle fibers behind allowing recurrence of symptoms. ${ }^{9}$ This is understandable as most of the commercially available staplers have 3 to $8 \mathrm{~mm}$ of noncutting anvil at the tip, which would theoretically allow preservation of the muscle. While some centers may have their stapler loads filed down to the cut edge to prevent this complication, this is unpractical and is the exception and not the norm.
When only the diverticular septum is divided regardless of the technique and device used, one does not close the mucosal defect and trusts the mediastinal planes to heal by secondary intention alone, thus increasing the risk of mediastinal leaks and other complications. ${ }^{10}$

Per oral CP myotomy allows precise division of all the muscle fibers and secure mucosal closure. One caveat of that is the diverticulum is left in place and not resected. Our experience and that of others is that when the myotomy is complete, the diverticulum empties easily and promptly into the esophageal lumen without causing any symptoms. It is plausible that a very large and deep diverticulum could benefit from a diverticulectomy.

Thoracic surgeons benefit from actively learning and evaluating emerging technology and endoscopic techniques for the management of esophageal diseases. Z-POEM may be performed by operators with third-space endoscopy skills and include those from either a surgical or gastroenterology background.

Long-term follow-up is critical to ensure the durability of this technique, and comparative data with other established techniques will be needed in this disease.

\section{Disclosures}

The authors have nothing to disclose.

Conflict of Interest

None declared.

\section{References}

1 Ludlow A. A case of obstructed deglutition from a preternatural dilatation of and bag formed in the pharynx. Med Observ Inq 1769;3:85-101

2 Mosher HP. Webs and pouches of the esophagus, their diagnosis and treatment. Surg Gynecol Obstet 1917;25:175-187

3 Stewart K, Sen P. Pharyngeal pouch management: an historical review. J Laryngol Otol 2016;130(02):116-120

4 Collard JM, Otte JB, Kestens PJ. Endoscopic stapling technique of esophagodiverticulostomy for Zenker's diverticulum. Ann Thorac Surg 1993;56(03):573-576

5 Weerda H, Pedersen P, Wehmer H, Braune H. A new laryngoscope for endolaryngeal microsurgery. A contribution to injector respiration (author's transl) [in German]. Arch Otorhinolaryngol 1979; 225(02):103-106

6 Mulder CJ, Costamagna G, Sakai P. Zenker's diverticulum: treatment using a flexible endoscope. Endoscopy 2001;33(11):991-997

7 Yang J, Novak S, Ujiki M, et al. An international study on the use of peroral endoscopic myotomy in the management of Zenker's diverticulum. Gastrointest Endosc 2020;91(01):163-168

8 Repici A, Spadaccini M, Belletrutti PJ, et al. Peroral endoscopic septotomy for short-septum Zenker's diverticulum. Endoscopy 2020;52(07):563-568

9 Bonavina L, Aiolfi A, Scolari F, Bona D, Lovece A, Asti E. Long-term outcome and quality of life after transoral stapling for Zenker diverticulum. World J Gastroenterol 2015;21(04):1167-1172

10 Bahsi S, Rustemova N, Vosoughi K, et al. Zenker's diverticulum peroral endoscopic myotomy using a scissors-type knife. Endoscopy 2019;51(08):E231-E232 